\title{
Pair creation in electric fields, anomalies, and renormalization of the electric current
}

\author{
Antonio Ferreiro* and Jose Navarro-Salas ${ }^{\dagger}$ \\ Departamento de Fisica Teorica and IFIC, Centro Mixto Universidad de Valencia-CSIC, \\ Facultad de Fisica, Universidad de Valencia, Burjassot-46100, Valencia, Spain
}

(Received 9 March 2018; published 14 June 2018)

\begin{abstract}
We investigate the Schwinger pair production phenomena in spatially homogeneous strong electric fields. We first consider scalar QED in four-dimensions and discuss the potential ambiguity in the adiabatic order assignment for the electromagnetic potential required to fix the renormalization subtractions. We argue that this ambiguity can be solved by invoking the conformal anomaly when both electric and gravitational backgrounds are present. We also extend the adiabatic regularization method for spinor QED in two-dimensions and find consistency with the chiral anomaly. We focus on the issue of the renormalization of the electric current $\left\langle j^{\mu}\right\rangle$ generated by the created pairs. We illustrate how to implement the renormalization of the electric current for the Sauter pulse.
\end{abstract}

DOI: 10.1103/PhysRevD.97.125012

\section{INTRODUCTION}

A time-dependent gravitational field yields the creation operators of quantum fields to evolve into a superposition of creation and annihilation operators. This produces the spontaneous creation of particle-antiparticle pairs [1-4]. This effect was first discovered and widely analyzed in the physical context of an expanding universe $[5,6]$. In the early seventies, a similar transformation between creation and annihilation operators was proved to occur for accelerated observers in the Rindler wedge of Minkowski space [7] and also in the spacetime describing a gravitational collapse forming a black hole [8]. Subsequent investigations concluded that gravitons are also created by the expansion of the universe $[9,10]$. Shortly after the proposal of the inflationary universe [11], the creation of scalar perturbations was analyzed in [12]. A similar superposition of creation and annihilation operators takes place if the quantized field is coupled to a time-varying scalar field background. Therefore, particle creation can be enhanced after the end of inflation, when the inflation, regarded as an external scalar field coupled to quantized matter fields, starts to rapidly oscillate in time [13].

In this paper we want to focus on the particle creation phenomena caused by a time varying background gauge

\footnotetext{
*antonio.ferreiro@ific.uv.es

jnavarro@ific.uv.es
}

Published by the American Physical Society under the terms of the Creative Commons Attribution 4.0 International license. Further distribution of this work must maintain attribution to the author(s) and the published article's title, journal citation, and DOI. Funded by SCOAP ${ }^{3}$. field. These electromagnetic processes are strongly motivated by the upcoming high intensity and ultrashort laser experiments $[14,15]$, which will allow us to understand nonperturbative regimes in QED where vacuum particle creation becomes relevant [16]. The most paradigmatic example of this is the pair-production by a strong and spatially homogeneous electric field $\vec{E}(t)=E(t) \hat{x}$, where one assumes a configuration with an initial vanishing electric field $E(t) \rightarrow 0$ as $t \rightarrow-\infty$, an intermediate period with a smoothly varying electric field, and a final decay to a zero electric field $E(t) \rightarrow 0$ when $t \rightarrow+\infty$. A prototype for this is the pulsed configuration $\vec{E}=\left(E_{0} \cosh ^{-2}\left(\omega_{0} t\right), 0,0\right)$. In the limit $\omega_{0} \rightarrow 0$ one recovers the constant electric field, which is actually an inherent assumption in the well-known Schwinger effect [17]. Schwinger's derivation, extending previous work by Sauter [18] and Heisenberg and Euler [19], is obtained within the effective action formalism in quantum electrodynamics (QED). It has been of great interest in theoretical research over many years [20-29] and it may be at the border of being experimentally verified.

A fundamental problem in gravitational processes is the calculation of the expectation values $\left\langle T_{\mu \nu}\right\rangle$, which, in addition to provide definite quantities for energy density, pressure, or radiation fluxes, would act as the proper source for the (semiclassical) Einstein's equations. The computations are involved, as we have to deal with ultraviolet (UV) divergences not present in Minkowski space. The corresponding subtractions needed for renormalization requires more sophisticated methods. Equivalently, in a time-dependent electric field the proper source of the Maxwell equations is the electric current which again possesses UV divergences. In this paper we analyze the renormalization of the electric current $\left\langle j_{\mu}\right\rangle$ for spatially homogeneous 
electric fields, like the pulsed configuration mentioned above, by importing and extending renormalization methods originally proposed in cosmological scenarios [30-36]. The electric current is probably the most important local observable to be consider in a near-future detection of the Schwinger effect. Therefore, the renormalization of both observables $\left\langle T_{\mu \nu}\right\rangle$ and $\left\langle j_{\mu}\right\rangle$ merit a detailed and simultaneous analysis. Moreover, new interests in cosmological scenarios of the Schwinger effect [37,38] demand the need of a regularization method involving both electric and gravitational fields.

The goal of this paper is to further extend the adiabatic regularization method for scalar [30-32] and Dirac fields [33-36] living in a Friedmann-Lemaitre-Robertson-Walker (FLRW) spacetime by adding the interaction with an external homogeneous electric field. The organization of the paper is as follows. In Sec. II we first consider scalar QED in four-dimensions and analyze the ambiguity in the adiabatic order assignment for the electromagnetic potential. We fix the ambiguity by invoking agreement with the trace anomaly when both electric and gravitational fields are present. In Sec. III we further extend the adiabatic scheme for Dirac fermions in two-dimensions. We find consistency with the axial anomaly. In Sec. IV we illustrate how to evaluate the renormalized electric current for the Sauter pulse in flat space using the improved renormalization methods introduced in Sec. III. Finally, in Sec. V we summarize our conclusions.

\section{SCALAR QED IN FLRW SPACETIME, ADIABATIC EXPANSION, AND RENORMALIZATION}

Let us consider a quantized charged scalar field in a FLRW metric of the form $d s^{2}=d t^{2}-a^{2}(t) d \vec{x}^{2}$. The scalar field obeys the Klein-Gordon field equation (we assume a generic coupling $\xi$ to the scalar curvature)

$$
\left(D_{\mu} D^{\mu}+m^{2}+\xi R\right) \phi=0,
$$

where

$$
D_{\mu} \phi=\left(\nabla_{\mu}+i q A_{\mu}\right) \phi .
$$

Assuming that the electric field is spatially homogeneous and the magnetic field is zero, we take the electric field in the direction of the $x$ axis. For our purposes it is very convenient to choose a gauge such that only the $x$-component of the vector potential is nonvanishing: $A_{\mu}=(0,-A(t), 0,0)$. Therefore, the field strength is given by $F_{0 i}=$ $(-\dot{A}(t), 0,0)$ and the Klein-Gordon equation becomes

$\ddot{\phi}-\frac{\vec{\nabla}^{2}}{a^{2}} \phi+3 \frac{\dot{a}}{a} \dot{\phi}+\frac{2 i q A}{a^{2}} \partial_{x} \phi+\frac{q^{2} A^{2}}{a^{2}} \phi+\left(m^{2}+\xi R\right) \phi=0$.
The quantized field is expanded in Fourier modes as

$\phi(x)=\frac{1}{\sqrt{2(2 \pi a)^{3}}} \int d^{3} \vec{k}\left[A_{\vec{k}} e^{i \vec{k} \vec{x}} h_{\vec{k}}(t)+B_{\vec{k}}^{\dagger} e^{-i \vec{k} \vec{x}} h_{-\vec{k}}^{*}(t)\right]$,

where $A_{\vec{k}}^{\dagger}, B_{\vec{k}}^{\dagger}$ and $A_{\vec{k}}, B_{\vec{k}}$ are the usual creation and annihilation operators. The mode functions $h_{\vec{k}}(t)$ must obey the Wronskian consistency condition

$$
h_{\vec{k}} \dot{h}_{\vec{k}}^{*}-h_{\vec{k}}^{*} \dot{h}_{\vec{k}}=2 i .
$$

Substituting (4) into (3) we get the equation

$$
\begin{aligned}
\ddot{h}_{\vec{k}} & +\left(m^{2}+\frac{\vec{k}^{2}}{a^{2}}-2 \frac{q A k_{x}}{a^{2}}+\frac{q^{2} A^{2}}{a^{2}}+\left(6 \xi-\frac{3}{4}\right) \frac{\dot{a}^{2}}{a^{2}}\right. \\
& \left.+\left(6 \xi-\frac{3}{2}\right) \frac{\ddot{a}}{a}\right) h_{\vec{k}}=0 .
\end{aligned}
$$

One can then write the vacuum expectation values for the stress-energy tensor as an integral over modes, producing an expression as

$$
\left\langle T^{\mu \nu}\right\rangle=\int d^{3} k T^{\mu \nu}(\vec{k}, t) .
$$

In general, the above integral is ultraviolet divergent. To renormalize (7), one should identify the subtraction terms that can serve to remove the parts of $T^{\mu \nu}(\vec{k}, t)$ which would diverge when integrated over $\vec{k}$. A physically motivated procedure for doing this is to perform an adiabatic expansion of the mode functions $h_{\vec{k}}(t)$, in powers of $a(t), \dot{a}(t), \ddot{a}(t)$, etc., and also in powers of $A(t), \dot{A}(t)$, $\ddot{A}(t)$, etc., around the free solutions. The mode expansion is then plugged in $T^{\mu \nu}(\vec{k}, t)$ to generate an adiabatic series. The minimal number of terms in this series are subtracted from $T^{\mu \nu}(\vec{k}, t)$ to cancel out all UV divergences. This method was first developed in the context of a scalar field living in spatially homogeneous cosmological backgrounds [30-32] and without any additional external field. For more recent studies see [33-36,39]. It was also applied, in the absence of gravity, to the pair-creation in a strong electric field scenario in [40-44]. Here we will reexamine the method when both the gravitational and the electric backgrounds are present. We will show that a consistent adiabatic order assignment is necessary for the method to agree with the combined conformal anomaly generated by the gravitational and the electromagnetic fields.

\section{A. Adiabatic expansion}

The adiabatic expansion for the scalar field modes is based on the usual WKB ansatz. For reasons to be explained later (see Sec. III) we shall not assume a priori the WKB ansatz. Instead, we will assume a most general ansatz by expanding the modes as follows 


$$
\begin{aligned}
h_{\vec{k}} & =H_{\vec{k}}(t) e^{-i \int^{t} \Omega_{\vec{k}}\left(t^{\prime}\right) d t^{\prime}}, \\
\Omega_{\vec{k}}(t) & =\omega_{\vec{k}}+\omega^{(1)}+\omega^{(2)}+\cdots, \\
H_{\vec{k}}(t) & =\frac{1}{\sqrt{\omega_{\vec{k}}}}+H_{\vec{k}}^{(1)}(t)+\cdots
\end{aligned}
$$

where $\omega_{\vec{k}}=\sqrt{m^{2}+\frac{\vec{k}^{2}}{a^{2}}}$ and $H_{\vec{k}}(t)$ and $\Omega_{\vec{k}}(t)$ are real functions. One can substitute the above ansatz into Eq. (6) and the Wronskian condition (5). We then get the equations (we drop the $\vec{k}$ index for simplicity)

$$
\begin{gathered}
\ddot{H}-H \Omega^{2}+\left[\omega^{2}-2 \frac{q A}{a} \frac{k_{x}}{a}+\frac{q^{2} A^{2}}{a^{2}}+\left(6 \xi-\frac{3}{4}\right) \frac{\dot{a}^{2}}{a^{2}}\right. \\
\left.+\left(6 \xi-\frac{3}{2}\right) \frac{\ddot{a}}{a}\right] H=0 \\
\Omega H^{2}=1 .
\end{gathered}
$$

We have to solve order by order to obtain the different terms of the expansion. As usual [1], we will consider $a(t)$ of adiabatic order zero, $\dot{a}(t)$ of adiabatic order one, etc. However, to get an unique series expansion we have to assign also an adiabatic order to the vector potential function $A(t)$. We will choose $A(t)$ to be of adiabatic order 1 . This assignment of adiabatic order 1 is consistent with the scaling dimension of the field $A(t)$, as it possesses the same dimensions as $\dot{a}$. The mass dimension of the scale

factor $a(t)$ is zero, while that of $\dot{a}(t)$, or the field $A(t)$, is unity. [We will reexamine this point in connection with the trace anomaly in subsection C.] Therefore, $\dot{A}(t)$ will be of adiabatic order $2, \ddot{A}(t)$ of order 3 and so on. The adiabatic order 0 coincides with the solution for $A(t)=0$ :

$$
\begin{gathered}
\omega^{(0)}=\omega \\
H^{(0)}=\frac{1}{\sqrt{\omega}},
\end{gathered}
$$

and hence

$$
h_{\vec{k}}^{(0)}=\frac{1}{\sqrt{m^{2}+\frac{\vec{k}^{2}}{a^{2}}}} e^{-i \int t \sqrt{m^{2}+\frac{\vec{k}^{2}}{a^{2}}} d t^{\prime}} .
$$

\section{First adiabatic order}

By keeping only terms of first adiabatic order in (9), the system of two equations gives

$$
\begin{aligned}
-2 \omega \omega^{(1)} H^{(0)}-\frac{2 q A k_{x}}{a^{2}} H^{(0)} & =0, \\
2 \omega H^{(0)} H^{(1)}+\omega^{(1)} H^{(0)} H^{(0)} & =0 .
\end{aligned}
$$

The solution is

$$
H^{(1)}=\frac{k_{x} q A}{2 a^{2} \omega^{5 / 2}}, \quad \omega^{(1)}=-\frac{k_{x} q A}{a^{2} \omega} .
$$

\section{Second adiabatic order}

In the same way, the second-order terms of (9) give

$$
\begin{aligned}
\ddot{H}^{(0)}-\left(2 \omega \omega^{(2)}+\left(\omega^{(1)}\right)^{2}+\frac{q^{2} A^{2}}{a^{2}}+\left(6 \xi-\frac{3}{4}\right) \frac{\dot{a}^{2}}{a^{2}}+\left(6 \xi-\frac{3}{2}\right) \frac{\ddot{a}}{a}\right) H^{(0)}+\left(-2 \omega^{(1)} \omega-\frac{2 q A k_{x}}{a^{2}}\right) H^{(1)} & =0, \\
\omega^{(2)}\left(H^{(0)}\right)^{2}+2 \omega^{(1)} H^{(0)} H^{(1)}+\omega\left(H^{(1)}\right)^{2}+2 \omega H^{(2)} H^{(0)} & =0,
\end{aligned}
$$

which has as solutions

$$
\begin{aligned}
& \omega^{(2)}=\frac{3 \xi \ddot{a}}{a \omega}-\frac{3 \ddot{a}}{4 a \omega}+\frac{3 \xi \dot{a}^{2}}{a^{2} \omega}-\frac{3 \dot{a}^{2}}{8 a^{2} \omega}-\frac{k_{x}^{2} q^{2} A^{2}}{2 a^{4} \omega^{3}}+\frac{q^{2} A^{2}}{2 a^{2} \omega}-\frac{\ddot{\omega}}{4 \omega^{2}}+\frac{3 \dot{\omega}^{2}}{8 \omega^{3}}, \\
& H^{(2)}=\frac{5 k_{x}^{2} q^{2} A^{2}}{8 a^{4} \omega^{9 / 2}}-\frac{q^{2} A^{2}}{4 a^{2} \omega^{5 / 2}}-\frac{3 \xi \ddot{a}}{2 a \omega^{5 / 2}}+\frac{3 \ddot{a}}{8 a \omega^{5 / 2}}-\frac{3 \xi \dot{a}^{2}}{2 a^{2} \omega^{5 / 2}}+\frac{3 \dot{a}^{2}}{16 a^{2} \omega^{5 / 2}}+\frac{\ddot{\omega}}{8 \omega^{7 / 2}}-\frac{3 \dot{\omega}^{2}}{16 \omega^{9 / 2}} .
\end{aligned}
$$

\section{Third and fourth adiabatic order}

The same procedure can be repeated for all higher orders. The third- and fourth-order terms of the expansion are explicitly written in Appendix A.

\section{B. Conformal anomaly}

We check now the consistency of our adiabatic expansion. A nontrivial test for our proposal is to reproduce the trace anomaly for the quantized charged scalar field for $\xi=1 / 6$ and $m=0$. To evaluate the trace anomaly in the adiabatic regularization method, we have to start with a massive field and take the massless limit at the end of the calculation. Moreover, for a massive charged field $T_{\mu}^{\mu}=2 m^{2} \phi \phi^{\dagger}$. However, this formal identification does not imply that $\left\langle T_{\mu}^{\mu}\right\rangle_{\text {ren }}=2 m^{2}\left\langle\phi \phi^{\dagger}\right\rangle_{\text {ren }}$. The divergences of the stress-energy tensor components have terms of fourth adiabatic order, while the divergences of $\left\langle\phi \phi^{\dagger}\right\rangle$ involve only terms till second adiabatic order. Therefore, in order to evaluate the trace anomaly by using the above formal expression, the adiabatic subtractions for $\left\langle\phi \phi^{\dagger}\right\rangle$ should also include subtractions up to fourth adiabatic order. The same 
argument has been used to work out the trace anomaly of a real scalar field [1]. Therefore,

$$
\left\langle T_{\mu}^{\mu}\right\rangle_{\mathrm{ren}}=\lim _{m \rightarrow 0} 2 m^{2}\left(\left\langle\phi \phi^{\dagger}\right\rangle_{\mathrm{ren}}-\left\langle\phi \phi^{\dagger}\right\rangle^{(4)}\right) .
$$

The fourth-order subtraction term, which produces a nonzero finite contribution when the mass vanishes, is codified in $\left\langle\phi \phi^{\dagger}\right\rangle^{(4)}$. The piece $m^{2}\left\langle\phi \phi^{\dagger}\right\rangle_{\text {ren }}$ vanishes when $m^{2} \rightarrow 0$. The remaining term produces the anomaly. We will now calculate this term.

Let us first consider the two-point function for our the complex scalar field

$$
\left\langle\phi \phi^{\dagger}\right\rangle=\frac{1}{2(2 \pi a)^{3}} \int\left|h_{\vec{k}}\right|^{2} d^{3} k,
$$

and, using the adiabatic expansion for the modes, one can evaluate the corresponding $n$-order adiabatic terms

$$
\begin{aligned}
\left\langle\phi(t) \phi^{\dagger}(t)\right\rangle^{(n)} & =\frac{1}{2(2 \pi a)^{3}} \int\left(\left|h_{\vec{k}}\right|^{2}\right)^{(n)} d^{3} k \\
& =\frac{2 \pi}{2(2 \pi a)^{3}} \int_{0}^{\infty} \int_{-\infty}^{\infty}\left|k_{\perp}\right|\left(\left|h_{\vec{k}}\right|^{2}\right)^{(n)} d k_{x} d k_{\perp},
\end{aligned}
$$

where we have chosen a preferred direction $k_{\|}=k_{x}$. After some computation, the trace anomaly is finally given by

$$
\begin{aligned}
\left\langle T_{\mu}^{\mu}\right\rangle_{\mathrm{ren}} & =\lim _{m^{2} \rightarrow 0}-2 m^{2}\left\langle\phi \phi^{\dagger}\right\rangle^{(4)} \\
& =\frac{a^{(4)}}{240 \pi^{2} a}+\frac{\ddot{a}^{2}}{240 \pi^{2} a^{2}}+\frac{a^{(3)} \dot{a}}{80 \pi^{2} a^{2}}-\frac{\dot{a}^{2} \ddot{a}}{80 \pi^{2} a^{3}}-\frac{q^{2} \dot{A}^{2}}{48 \pi^{2} a^{2}} .
\end{aligned}
$$

This last term is in full agreement with the well-known trace anomaly for a background electromagnetic field in Minkowski spacetime [45]. The remaining terms reproduce the trace anomaly of the gravitational FLRW background. The result is twice the value obtained for a real scalar field [1]. In covariant form, we get

$$
\begin{aligned}
\left\langle T_{\mu}^{\mu}\right\rangle_{\mathrm{ren}}= & \frac{1}{1440 \pi^{2}}\left\{\square R-\left(R^{\mu \nu} R_{\mu \nu}-\frac{1}{3} R^{2}\right)\right\} \\
& +\frac{q^{2}}{96 \pi^{2}} F_{\mu \nu} F^{\mu \nu} .
\end{aligned}
$$

The ability to reproduce the conformal anomaly is a nontrivial test for our renormalization scheme.

\section{Discussion on the adiabatic order assignment}

In most references in the literature [37,38,40-44] the vector potential is assumed of adiabatic order 0 , which differs from our previous assumption. This means that the adiabatic expansion proposed here and its physical consequences will be potentially different from those considered in the previous literature on this topic. Assuming $A(t)$ of adiabatic order zero, the leading adiabatic order of the modes would be

$\tilde{h}_{\vec{k}}^{(0)}=\frac{1}{\sqrt{m^{2}+\frac{\vec{k}^{2}}{a^{2}}-\frac{2 q A k_{x}}{a^{2}}+\frac{q^{2} A^{2}}{a^{2}}}} e^{-i \int t \sqrt{m^{2}+\frac{\vec{k}^{2}}{a^{2}}-\frac{2 q A k_{x}}{a^{2}}+\frac{q^{2} A^{2}}{a^{2}}} d t^{\prime}}$.

A natural question now is to investigate whether this alternative adiabatic order assignment, is also able to reproduce the trace anomaly. The adiabatic order of the modes is given in Appendix B. When gravity is included, the result for the trace anomaly is problematic. Performing the adiabatic subtraction for the stress-energy tensor until the fourth adiabatic order, as usual for quantized fields interacting with gravity [1,4,30-32], one gets for the trace anomaly $\left\langle T_{\mu}^{\mu}\right\rangle_{\text {ren }}=\lim _{m^{2} \rightarrow 0}-2 m^{2}\left\langle\phi \phi^{\dagger}\right\rangle^{(4)}$. The result is

$$
\begin{aligned}
\left\langle T_{\mu}^{\mu}\right\rangle_{\text {ren }}= & \frac{1}{1440 \pi^{2}}\left\{\square R-\left(R^{\mu \nu} R_{\mu \nu}-\frac{1}{3} R^{2}\right)\right\} \\
& -\frac{q^{2} \ddot{a} \dot{A}^{2}}{90 \pi^{2} m^{2} a^{3}}-\frac{7 q^{2} \dot{a}^{2} \dot{A}^{2}}{1440 \pi^{2} m^{2} a^{4}}-\frac{q^{2} \dot{a} \dot{A} \ddot{A}}{720 \pi^{2} m^{2} a^{3}} \\
& +\frac{q^{2} \ddot{A}^{2}}{160 \pi^{2} m^{2} a^{2}}-\frac{7 q^{4} \dot{A}^{4}}{1440 \pi^{2} m^{4} a^{4}}+\frac{q^{2} A^{(3)} \dot{A}}{120 \pi^{2} m^{2} a^{2}}
\end{aligned}
$$

The terms involving $A(t)$ and their derivatives do not reproduce the expected contribution to the trace anomaly $\frac{q^{2}}{96 \pi^{2}} F_{\mu \nu} F^{\mu \nu}$. In fact these terms are infrared divergent as $m \rightarrow 0$. To reproduce the electromagnetic piece of the trace anomaly (which is then of second adiabatic order), one is forced to remove gravity (i.e., take $a(t)=1$ ) and renormalize the stress-energy tensor until the second adiabatic order only. This way one would obtain

$$
\left\langle T_{\mu}^{\mu}\right\rangle_{\mathrm{ren}}^{a=1}=-2 \lim _{m^{2} \rightarrow 0} m^{2}\left\langle\phi \phi^{\dagger}\right\rangle^{(2)}=\frac{q^{2}}{96 \pi^{2}} F_{\mu \nu} F^{\mu \nu} .
$$

If one ignores the gravitational background and take $a(t)=1$, it is then perfectly consistent to choose $A(t)$ of adiabatic order zero. However, if one includes gravity the renormalization with the zeroth adiabatic order assignment for $A(t)$ seems to be not consistent with the trace anomaly. 
This is an important novelty of this paper and we will go back to this point when consider the trace anomaly of Dirac fermions.

\section{SPINOR QED IN TWO DIMENSIONS, ADIABATIC EXPANSION, AND RENORMALIZATION}

In two dimensions, the Dirac equation in presence of an external homogeneous electric field and a background metric of the FLRW form $d s^{2}=d t^{2}-a^{2}(t) d x^{2}$ is

$$
\left(i \underline{\gamma}^{\mu} \nabla_{\mu}-m\right) \psi=0
$$

where $\nabla_{\mu} \equiv \partial_{\mu}-\Gamma_{\mu}-i q A_{\mu}$ and $\Gamma_{\mu}$ is the spin connection. $\gamma^{\mu}(x)$ are the spacetime-dependent Dirac matrices satisfy-

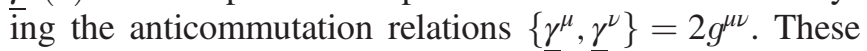
gamma matrices are related with the Minkowskian ones by $\underline{\gamma}^{0}(t)=\gamma^{0}$ and $\underline{\gamma}^{1}(t)=\gamma^{1} / a(t)$, and the components of the spin connections are $\Gamma_{0}=0$ and $\Gamma_{1}=(\dot{a} / 2) \gamma_{0} \gamma_{1}$. Therefore, $\underline{\gamma}^{\mu} \Gamma_{\mu}=-\frac{\dot{a}}{2 a} \gamma_{0}$ and we fix a gauge for the potential as $A_{\mu}=(0,-A(t))$. The Dirac equation (25) becomes

$$
\left(i \gamma^{0} \partial_{0}+\frac{i}{2} \frac{\dot{a}}{a} \gamma^{0}+\left(\frac{i}{a} \partial_{1}+\frac{q A_{1}}{a}\right) \gamma^{1}-m\right) \psi=0
$$

From now on we will use the Weyl representation (with $\left.\gamma^{5} \equiv \gamma^{0} \gamma^{1}\right)$

$\gamma^{0}=\left(\begin{array}{ll}0 & 1 \\ 1 & 0\end{array}\right), \quad \gamma^{1}=\left(\begin{array}{cc}0 & 1 \\ -1 & 0\end{array}\right), \quad \gamma^{5}=\left(\begin{array}{cc}-1 & 0 \\ 0 & 1\end{array}\right)$.

Expanding the field in momentum modes $\psi(t, x)=$ $\sum_{k} \psi_{k}(t) e^{i k x}$, Eq. (26) is converted into

$$
\left(\partial_{0}+\frac{\dot{a}}{2 a}+\frac{i}{a}(k+q A) \gamma^{5}+i m \gamma^{0}\right) \psi_{k}=0
$$

We can construct two independent spinor solutions

$$
\begin{aligned}
& u_{k}(t, x)=\frac{e^{i k x}}{\sqrt{2 \pi a}}\left(\begin{array}{c}
h_{k}^{I}(t) \\
-h_{k}^{I I}(t)
\end{array}\right) \\
& v_{k}(t, x)=\frac{e^{-i k x}}{\sqrt{2 \pi a}}\left(\begin{array}{c}
h_{-k}^{I I *}(t) \\
h_{-k}^{I *}(t)
\end{array}\right),
\end{aligned}
$$

where $h_{k}^{I}(t)$ and $h_{k}^{I I}(t)$ are appropriate solutions of the equations

$$
\dot{h}_{k}^{I}-\frac{i}{a}(k+q A) h_{k}^{I}-i m h_{k}^{I I}=0
$$

$$
\dot{h}_{k}^{I I}+\frac{i}{a}(k+q A) h_{k}^{I I}-i m h_{k}^{I}=0 .
$$

The normalization condition $\left|h^{I}\right|^{2}+\left|h^{I I}\right|^{2}=1$ leads to the usual Dirac scalar products

$$
\begin{gathered}
\left(u_{k}, u_{k^{\prime}}\right)=\int d x a u_{k}^{\dagger} u_{k^{\prime}}=\delta\left(k-k^{\prime}\right) \\
\left(v_{k}, v_{k^{\prime}}\right)=\int d x a v_{k}^{\dagger} v_{k^{\prime}}=\delta\left(k-k^{\prime}\right) \\
\left(u_{k}, v_{k^{\prime}}\right)=\int d x a u_{k}^{\dagger} v_{k^{\prime}}=0 .
\end{gathered}
$$

This condition guarantees the anticommutation relations for the creation and annihilation operators $B_{k}$ and $D_{k}$, defined by the expansion of the Dirac field operator in terms of the above spinors

$$
\psi(t, x)=\int d k\left[B_{k} u_{k}(t, x)+D_{k}^{\dagger} v_{k}(t, x)\right] .
$$

The usual equal-time anticommutation relation holds

$$
\left\{\psi_{\alpha}(t, x), \psi_{\beta}^{\dagger}(t, y)\right\}=\delta(x-y) \delta_{\alpha \beta} .
$$

\section{A. Adiabatic expansion}

As explained above, the Dirac equation splits into a system of two coupled equation for $h_{k}^{I}$ and $h_{k}^{I I}$. Our aim here is to obtain a self-consistent adiabatic expansion for these two functions $h_{k}^{I}$ and $h_{k}^{I I}$. Inspired by the adiabatic expansion for Dirac fermions in a four-dimensional FLRW spacetime [33-36], we propose the following ansatz (for simplicity in the notation we omit the index $k$ in the functions $F_{k}, G_{k}$ and $\Omega_{k}$ )

$$
\begin{gathered}
h_{k}^{I}=\sqrt{\frac{\omega-k / a}{2 \omega}} F(t) e^{-i \int^{t} \Omega\left(t^{\prime}\right) d t^{\prime}} \\
h_{k}^{I I}=-\sqrt{\frac{\omega+k / a}{2 \omega}} G(t) e^{-i \int^{t} \Omega\left(t^{\prime}\right) d t^{\prime}},
\end{gathered}
$$

where $\omega=\sqrt{m^{2}+k^{2} / a^{2}}$. We find 


$$
\begin{gathered}
(\omega-k / a)\left(\dot{F}-i \Omega F-\frac{i}{a}(k+q A) F\right)+\frac{k}{a} \frac{m^{2}}{2 \omega^{2}} \frac{\dot{a}}{a} F+i m^{2} G=0 \\
(\omega+k / a)\left(\dot{G}-i \Omega G+\frac{i}{a}(k+q A) G\right) \\
-\frac{k}{a} \frac{m^{2}}{2 \omega^{2}} \frac{\dot{a}}{a} G+i m^{2} F=0
\end{gathered}
$$$$
(\omega-k / a)\left(F^{(1)}+F^{(1) *}\right)+(\omega+k / a)\left(G^{(1)}+G^{(1) *}\right)=0 .
$$

and the normalization condition

$$
\frac{\omega+k / a}{2 \omega}|G|^{2}+\frac{\omega-k / a}{2 \omega}|F|^{2}=1 .
$$

As in previous sections, we have also assumed that $A(t)$ is of adiabatic order 1 . The zeroth adiabatic order solution is the Minkowskian solution with vanishing electric field

$$
\begin{gathered}
h^{I(0)}=\sqrt{\frac{\omega-k / a}{2 \omega}} e^{-i t \omega} \\
h^{I I(0)}=-\sqrt{\frac{\omega+k / a}{2 \omega}} e^{-i t \omega}
\end{gathered}
$$

We expand the functions $F, G, \Omega$ adiabatically

$$
\begin{aligned}
& F=1+F^{(1)}+F^{(2)}+\cdots, \\
& G=1+G^{(1)}+G^{(2)}+\cdots, \\
& \Omega=\omega+\omega^{(1)}+\omega^{(2)}+\cdots,
\end{aligned}
$$

We recursively calculate order by order the higher-order adiabatic terms. We split the functions $F^{(n)}$ and $G^{(n)}$ into real and imaginary parts: $F^{(n)}=F_{x}^{(n)}+i F_{y}^{(n)}, G^{(n)}=G_{x}^{(n)}+i G_{y}^{(n)}$. The results up to second order are as follows.

\section{First adiabatic order}

By keeping only terms of first adiabatic order in (41), the system of three equations gives

$$
\begin{aligned}
& (\omega-k / a)\left(-i \omega F^{(1)}-i \omega^{(1)}-\frac{i}{a} k F^{(1)}-\frac{i}{a} q A\right) \\
& +\frac{k}{a} \frac{m^{2}}{2 \omega^{2}} \frac{\dot{a}}{a}+i m^{2} G^{(1)}=0 \\
& (\omega+k / a)\left(-i \omega G^{(1)}-i \omega^{(1)}+\frac{i}{a} k G^{(1)}+\frac{i}{a} q A\right) \\
& -\frac{k}{a} \frac{m^{2}}{2 \omega^{2}} \frac{\dot{a}}{a}+i m^{2} F^{(1)}=0
\end{aligned}
$$

$(\omega+k / a)\left(-\omega G_{x}^{(1)}-\omega^{(1)}+\frac{1}{a} k G_{x}^{(1)}+\frac{1}{a} q A\right)+m^{2} F_{x}^{(1)}=0$

$$
(\omega-k / a)\left(2 F_{x}^{(1)}\right)+(\omega+k / a)\left(2 G_{x}^{(1)}\right)=0 .
$$

The solution is

$$
\begin{gathered}
F_{x}^{(1)}=-\frac{q A(\omega+k / a)}{2 a \omega^{2}}, \quad G_{x}^{(1)}=\frac{q A(\omega-k / a)}{2 a \omega^{2}}, \\
\omega^{(1)}=\frac{k q A}{a^{2} \omega} .
\end{gathered}
$$

On the other hand, the real part of the system gives

$$
\begin{aligned}
& (\omega-k / a)\left(\omega F_{y}^{(1)}+\frac{1}{a} k F_{y}^{(1)}\right)+\frac{k}{a} \frac{m^{2}}{2 \omega^{2}} \frac{\dot{a}}{a}-m^{2} G_{y}^{(1)}=0 \\
& (\omega+k / a)\left(\omega G_{y}^{(1)}-\frac{1}{a} k G_{y}^{(1)}\right)-\frac{k}{a} \frac{m^{2}}{2 \omega^{2}} \frac{\dot{a}}{a}-m^{2} F_{y}^{(1)}=0 .
\end{aligned}
$$

These two equations are not independent. The obtained solution is

$$
F_{y}^{(1)}=M(t)-\frac{k \dot{a}}{2 a^{2} \omega^{2}}, \quad G_{y}^{(1)}=M(t),
$$

where $M(t)$ is an arbitrary first-order adiabatic function. We have checked that physical expectation values of local observables are independent to any potential ambiguity in the choice for $M(t)$. For simplicity we choose

$$
M(t)=\frac{k \dot{a}}{4 a^{2} \omega^{2}},
$$

and then

$$
F_{y}^{(1)}=-\frac{k \dot{a}}{4 a^{2} \omega^{2}}=-G_{y}^{(1)} .
$$




\section{Second adiabatic order}

In the same way, we solve the second-order terms of (41) and find

$$
\begin{aligned}
& F_{x}^{(2)}=\frac{A^{2} k q^{2}}{2 a^{3} \omega^{3}}-\frac{5 A^{2} m^{2} q^{2}}{8 a^{2} \omega^{4}}+\frac{A^{2} q^{2}}{2 a^{2} \omega^{2}}-\frac{k \ddot{a}}{8 a^{2} \omega^{3}}+\frac{m^{2} \ddot{a}}{8 a \omega^{4}}-\frac{\ddot{a}}{8 a \omega^{2}}+\frac{5 k m^{2} \dot{a}^{2}}{16 a^{3} \omega^{5}}-\frac{k \dot{a}^{2}}{16 a^{3} \omega^{3}}-\frac{5 m^{4} \dot{a}^{2}}{16 a^{2} \omega^{6}}+\frac{13 m^{2} \dot{a}^{2}}{32 a^{2} \omega^{4}}-\frac{3 \dot{a}^{2}}{32 a^{2} \omega^{2}}, \\
& G_{x}^{(2)}=\frac{A^{2} k q^{2}}{2 a^{3} \omega^{3}}-\frac{5 A^{2} m^{2} q^{2}}{8 a^{2} \omega^{4}}-\frac{A^{2} q^{2}}{2 a^{2} \omega^{2}}+\frac{k \ddot{a}}{8 a^{2} \omega^{3}}+\frac{m^{2} \ddot{a}}{8 a \omega^{4}}-\frac{\ddot{a}}{8 a \omega^{2}}-\frac{5 k m^{2} \dot{a}^{2}}{16 a^{3} \omega^{5}}+\frac{k \dot{a}^{2}}{16 a^{3} \omega^{3}}-\frac{5 m^{4} \dot{a}^{2}}{16 a^{2} \omega^{6}}+\frac{13 m^{2} \dot{a}^{2}}{32 a^{2} \omega^{4}}-\frac{3 \dot{a}^{2}}{32 a^{2} \omega^{2}}, \\
& \omega^{(2)}=-\frac{m^{2} \ddot{a}}{4 a \omega^{3}}+\frac{\ddot{a}}{4 a \omega}+\frac{5 m^{4} \dot{a}^{2}}{8 a^{2} \omega^{5}}-\frac{5 m^{2} \dot{a}^{2}}{8 a^{2} \omega a^{3}}+\frac{m^{2} q^{2} A^{2}}{2 a^{2} \omega^{3}},
\end{aligned}
$$$$
F_{y}^{(2)}=N(t)-\frac{5 m^{2} q A \dot{a}}{4 a^{2} \omega^{4}}+\frac{3 q A \dot{a}}{4 a^{2} \omega^{2}}+\frac{q \dot{A}}{2 a \omega^{2}},
$$$$
G_{y}^{(2)}=N(t) .
$$

Again we have the same ambiguity for $N(t)$. We choose for simplicity:

$$
N(t)=\frac{5 m^{2} q A \dot{a}}{8 a^{2} \omega^{4}}-\frac{3 q A \dot{a}}{8 a^{2} \omega^{2}}-\frac{q \dot{A}}{4 a \omega^{2}} .
$$

\section{B. Chiral anomaly}

To test the self-consistency of the above adiabatic expansion we are going to show how the chiral anomaly is obtained from it. We will consider the axial current

$$
j_{A}^{\mu}=\bar{\psi} \gamma^{\mu} \gamma^{5} \psi
$$

which is conserved in the massless limit. To evaluate the expectation value $\left\langle\nabla_{\mu} j_{A}^{\mu}\right\rangle$ we will reintroduce the mass and evaluate the right-hand-side of

$$
\left\langle\nabla_{\mu} j_{A}^{\mu}\right\rangle=2 i m\left\langle\bar{\psi} \gamma^{5} \psi\right\rangle
$$

in the limit $m \rightarrow 0$. Since the formal expression for $\left\langle\nabla_{\mu} j_{A}^{\mu}\right\rangle$ has divergences till second adiabatic order for a generic metric we need to perform subtractions in $\left\langle\bar{\psi} \gamma^{5} \psi\right\rangle$ up to second adiabatic order. Therefore,

$$
\left\langle\nabla_{\mu} j_{A}^{\mu}\right\rangle_{\text {ren }}=-\lim _{m \rightarrow 0} 2 i m\left\langle\bar{\psi} \gamma^{5} \psi\right\rangle^{(2)} .
$$

By writing $\left\langle\nabla_{\mu} j_{A}^{\mu}\right\rangle$ in terms of $\left\{h^{I}, h^{I I}\right\}$

$$
\left\langle\bar{\psi} \gamma^{5} \psi\right\rangle=\frac{1}{2 \pi a} \int_{-\infty}^{\infty} d k\left(h^{I I *} h^{I}-h^{I *} h^{I I}\right),
$$

and using our adiabatic series expansion, we arrive at

$$
\begin{aligned}
\left\langle\bar{\psi} \gamma^{5} \psi\right\rangle^{(2)}= & \frac{1}{2 \pi a} \int_{-\infty}^{\infty} d k \frac{i m}{\omega}\left(F_{y}^{(2)}-G_{y}^{(2)}+F_{y}^{(1)} G_{x}^{(1)}\right. \\
& \left.-G_{y}^{(1)} F_{x}^{(1)}\right)=\frac{i q \dot{A}}{2 \pi a m} .
\end{aligned}
$$

This result leads immediately to the axial anomaly in two dimensions

$$
\left\langle\nabla_{\mu} j_{A}^{\mu}\right\rangle_{\mathrm{ren}}=\frac{q \dot{A}}{a \pi}=-\frac{q}{2 \pi} \varepsilon^{\mu \nu} F_{\mu \nu}
$$

where $\varepsilon^{01}=|g|^{-1 / 2}=a^{-1}$. This result reproduces exactly the chiral anomaly for spinor $\mathrm{QED}_{2}[1,46]$. For a massive field we obtain $\left\langle\nabla_{\mu} j_{A}^{\mu}\right\rangle_{\text {ren }}=-\frac{q}{2 \pi} \varepsilon^{\mu \nu} F_{\mu \nu}+2 i m\left\langle\bar{\psi} \gamma^{5} \psi\right\rangle_{\text {ren }}$. The axial anomaly in two dimensions is the hallmark of the particle creation process caused by the electric field [47]. A manifestation of a similar phenomenon for photons in a gravitational scenario has been pointed out in [48].

\section{Conformal anomaly}

It is very easy to see how the method accounts for the trace anomaly. The trace of the energy momentum tensor can be written as:

$$
T_{\mu}^{\mu}=m \bar{\psi} \psi
$$

After renormalization we have a residual contribution when the mass goes to zero

$$
\left\langle T_{\mu}^{\mu}\right\rangle_{\mathrm{ren}}=\lim _{m \rightarrow 0}-m\langle\bar{\psi} \psi\rangle^{(2)} .
$$

By using the adiabatic expansion we can write:

$$
\begin{aligned}
\langle\bar{\psi} \psi\rangle^{(2)} & =\frac{1}{2 \pi a} \int_{-\infty}^{\infty} d k\left(h^{I *} h^{I I}+h^{I I *} h^{I}\right)^{(2)} \\
& =\frac{-1}{2 \pi a} \int_{-\infty}^{\infty} d k \frac{m}{\omega}\left(F_{x}^{(2)}+G_{x}^{(2)}+F_{x}^{(1)} G_{x}^{(1)}+F_{y}^{(1)} G_{y}^{(1)}\right)
\end{aligned}
$$

After integrating the corresponding adiabatic terms:

$$
\langle\bar{\psi} \psi\rangle^{(2)}=\frac{\ddot{a}}{12 \pi a m} .
$$

By using (70) we get

$$
\left\langle T_{\mu}^{\mu}\right\rangle_{\mathrm{ren}}=-\frac{\ddot{a}}{12 \pi a}=-\frac{R}{24 \pi},
$$


where in the last step we have used the expression of the two-dimensional scalar curvature in the terms of the expansion factor. The result agrees with the value of the trace anomaly for a Dirac spinor in two dimensions [49], which in turn coincides with the trace anomaly of a real scalar field $[4,50,51]$.

We would like to stress that, if one assumes the zeroth adiabatic order for $A(t)$, the result for the trace anomaly is the following

$$
\left\langle T_{\mu}^{\mu}\right\rangle_{\mathrm{ren}}=\lim _{m \rightarrow 0}\left[-\frac{\ddot{a}}{12 \pi a}-\frac{q^{2} \dot{A}^{2}}{6 \pi m^{2} a^{2}}\right]
$$

We find again a very unpleasant result, like (23) for scalar fields in four-dimensions. In the massless limit the above quantity is divergent and does not match the expected result. By contrast, the adiabatic order one for $A$ gives the right result (73) for the conformal anomaly.

\section{RENORMALIZED CURRENT FOR A PULSED ELECTRIC FIELD IN SPINOR QED}

\section{A. Vacuum choice for the Sauter pulse}

We consider now the Sauter pulse in a two-dimensional Minkowski space. It is driven by the electric field $E=$ $E_{0} \cosh ^{-2}\left(\omega_{0} t\right)$, with potential $A(t)=-\frac{E_{0}}{\omega_{0}}\left(\tanh \left(\omega_{0} t\right)+1\right)$. From the Dirac equation we find the coupled equations:

$$
\begin{gathered}
\dot{h}^{I}-i P h^{I}-i m h^{I I}=0 \\
\dot{h}^{I I}+i P h^{I I}-i m h^{I}=0,
\end{gathered}
$$

where we have defined $P=k+q A(t)$. One can decouple these equations and obtain

$$
\begin{gathered}
\left(\partial_{t}^{2}-i \dot{P}+P^{2}+m^{2}\right) h^{I}=0 \\
\left(\partial_{t}^{2}+i \dot{P}+P^{2}+m^{2}\right) h^{I I}=0 .
\end{gathered}
$$

The solution to the above equations, with the appropriated boundary conditions at $t=-\infty$, are given in terms of hypergeometric functions [52-54]

$$
\begin{gathered}
h^{I}=\sqrt{\frac{\omega-k}{2 \omega}} F(a, b, c ; \tau) \tau^{\alpha}(1-\tau)^{\beta} \\
h^{I I}=-\sqrt{\frac{\omega+k}{2 \omega}} F\left(a^{\prime}, b^{\prime}, c ; \tau\right) \tau^{\alpha}(1-\tau)^{\beta},
\end{gathered}
$$

where $\tau=1 / 2\left(1+\tanh \left(\omega_{0} t\right)\right), a=-i q \frac{E_{0}}{\omega^{2}}+\alpha+\beta, b=$ $1+i q \frac{E_{0}}{\omega^{2}}+\alpha+\beta, c=1+2 \alpha$ and

$$
\begin{aligned}
& \alpha=-\frac{i}{2 \omega_{0}} \sqrt{k^{2}+m^{2}}, \\
& \beta=\frac{i}{2 \omega_{0}} \sqrt{\left(k-2 \frac{q E_{0}}{\omega_{0}}\right)^{2}+m^{2}} .
\end{aligned}
$$

$a^{\prime}$ and $b^{\prime}$ are obtained from $a, b$ by replacing $E_{0} \rightarrow-E_{0}$ and $k \rightarrow-k$. In the limit $t \rightarrow-\infty$ or $\tau \rightarrow 0$, both solutions have the asymptotic behavior corresponding to positive frequency modes

$$
h^{I / I I} \sim \mp \sqrt{\frac{\omega \pm k}{2 \omega}} e^{-i \omega t} .
$$

\section{B. Renormalization of the electric current}

The two-dimensional electric current defined as the source of the Maxwell equation:

$$
\partial_{\mu} F^{\mu \nu}=j^{\nu}
$$

is $j^{\mu}=-q \bar{\psi} \gamma^{\mu} \psi$. The formal expectation values $\left\langle j^{\mu}\right\rangle$, expressed in terms of $h^{I}, h^{I I}$, are given by

$$
\begin{aligned}
& \left\langle j^{0}\right\rangle=-\frac{q}{2 \pi} \int_{-\infty}^{\infty} d k\left(\left|h^{I}\right|^{2}+\left|h^{I I}\right|^{2}\right) \\
& \left\langle j^{x}\right\rangle=-\frac{q}{2 \pi} \int_{-\infty}^{\infty} d k\left(\left|h^{I}\right|^{2}-\left|h^{I I}\right|^{2}\right) .
\end{aligned}
$$

For the 0-component it is easy to see that after renormalization and taking into account the normalization condition, it vanishes $\left\langle j^{0}\right\rangle_{\text {ren }}=0$. In order to renormalize the nonvanishing component of the electric current, the zeroth and first adiabatic orders need to be subtracted, i.e.,

$$
\begin{aligned}
\left\langle j^{x}\right\rangle_{\text {ren }}= & \frac{q}{2 \pi} \int_{-\infty}^{\infty} d k\left[\left|h^{I I}\right|^{2}-\left|h^{I}\right|^{2}-\left(\left|h^{I I}\right|^{2}-\left|h^{I}\right|^{2}\right)^{(0)}\right. \\
& \left.-\left(\left|h^{I I}\right|^{2}-\left|h^{I}\right|^{2}\right)^{(1)}\right] .
\end{aligned}
$$

After some calculations we find

$\left\langle j^{x}\right\rangle_{\mathrm{ren}}=\frac{q}{2 \pi} \int_{-\infty}^{\infty} d k\left(\left|h^{I I}\right|^{2}-\left|h^{I}\right|^{2}-\frac{k}{\omega}-\frac{q m^{2}}{\omega^{3}} A\right)$.

For simplicity we assume $q>0$. The above integral is finite and one can estimate it easily by numerical integration (see Fig. 1). We find a consistent behavior for $\left\langle j^{x}\right\rangle_{\text {ren. }}$. The induced current appears once the pulse starts and it depends on the values of the external parameters. $E_{c} \equiv m^{2} / q$ is the critical Schwinger value for pair production. Note that for $\omega_{0} \rightarrow 0$, i.e., the constant electric field configuration of the traditional Schwinger effect, $\left\langle j^{x}\right\rangle_{\text {ren }} \rightarrow \infty$. We will interpret this behavior later on. 


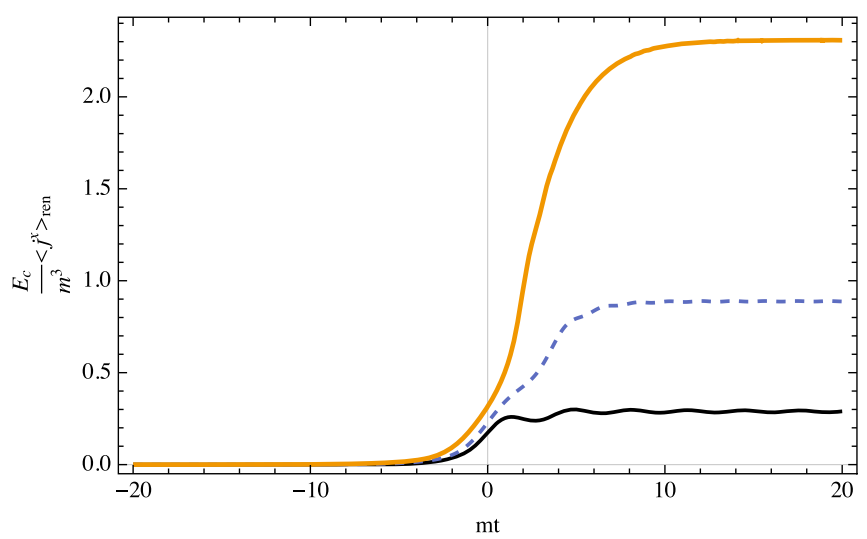

FIG. 1. Renormalized electric current (87) $\langle j x\rangle_{\text {ren }}$ for $E_{0}=2 E_{c}$ and $\omega_{0}=0.25 \mathrm{~m}$ (thick line), $\omega_{0}=0.35 \mathrm{~m}$ (dashed line) and $\omega_{0}=$ $0.5 m$ (black line) in natural units.

\section{Adiabatic regime and particle creation}

Let us consider the adiabatic expansion for the renormalized electric current itself. In this case, after subtracting the zeroth and first adiabatic order, and assuming an adiabatic configuration for $A(t)$, we find:

$\left\langle j^{x}\right\rangle_{\text {ren }}=\left\langle j^{x}\right\rangle^{(3)}+O^{(n>3)}=-\frac{7 q^{4} A^{3}}{30 m^{2} \pi}-\frac{q^{2} \ddot{A}}{6 m^{2} \pi}+O^{(n>3)}$

where $O^{(n>3)}$ are terms with adiabatic order higher than 3 . We note first that, assuming that at early times the electric configuration is of the form $E(t) \geq 0$ and $\dot{E}(t)>0$, and taking into account that

$$
A(t)=-\int_{-\infty}^{t} d t^{\prime} E\left(t^{\prime}\right)
$$

the renormalized current always takes positive values $\left\langle j^{x}\right\rangle_{\text {ren }}>0$ at early times, irrespective of the quantum state.

We can also compare the above expansion with the exact result for the electric current for the Sauter pulse

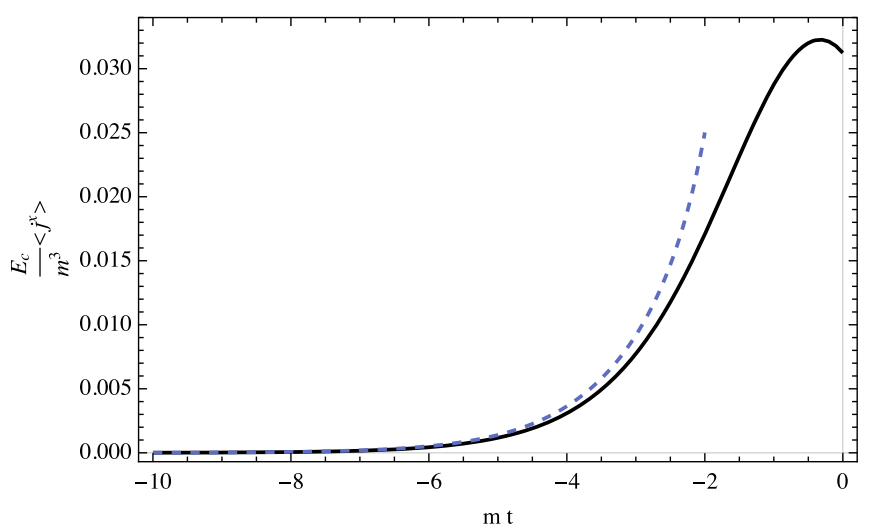

FIG. 2. Renormalized electric current (87) $\left\langle j^{x}\right\rangle_{\text {ren }}$ and third adiabatic order approximation (88) $\left\langle j_{x}\right\rangle^{(3)}$ for $E_{0}=E_{c}$ and $\omega_{0}=$ $0.5 m$ in natural units. (see Fig. 2). We observe that the configuration $A(t)$ defined by the Sauter pulse is only adiabatic at early times, and ceases to be adiabatic around the instant $m t \approx-4$, time before reaching the critical electric field needed for pair production. The non-adiabatic period extends to all times in the (Schwinger) limit $\omega_{0} \rightarrow 0$, and particles are created without end. Hence $\left\langle j^{x}\right\rangle_{\text {ren }}$ diverges, as note before. [Note that, in contrast, a configuration of the form $A(t)=$ $-E_{0}\left(\tanh \left(\omega_{0} t\right)+1\right)$, which corresponds to the electric field $E=E_{0} \omega_{0}$, is adiabatic for $\omega_{0} \rightarrow 0$ and the mean particle number $\langle N\rangle$ would be zero, in agreement with the adiabatic theorem].

\section{Backreaction}

An important effect due to particle creation is the backreaction of the current production into the former electric field. Let us briefly illustrate this effect within our approach for renormalizing the electric current. In the case of the Sauter pulse the induced electric current behaves as a smooth function and we can mimic it with a simple analytic function. Let us consider the pulse with $\omega_{0}=0.5 \mathrm{~m}$ and $E_{0}=4 E_{c}$. In this case we found that the electric current can be parametrize by a smooth function (see Fig. 3) such as:

$$
\left\langle j^{x}\right\rangle_{\text {ren }}(t)=\frac{K m^{3}}{E_{c}}(1+\tanh (K m t))
$$

where in this case $K=0.75$. The induced electric current also acts as source of the electromagnetic field according to the semiclassical Maxwell equations:

$$
\partial_{\mu} F^{\mu \nu}=\left\langle j^{\nu}\right\rangle_{\text {ren }}
$$

In our case this translates into $\ddot{A}(t)=\left\langle j^{x}\right\rangle_{\text {ren }}(t)$. We can easily integrate this equation and find the backreaction contribution to the electric field $E(t)=-\dot{A}(t)$ :

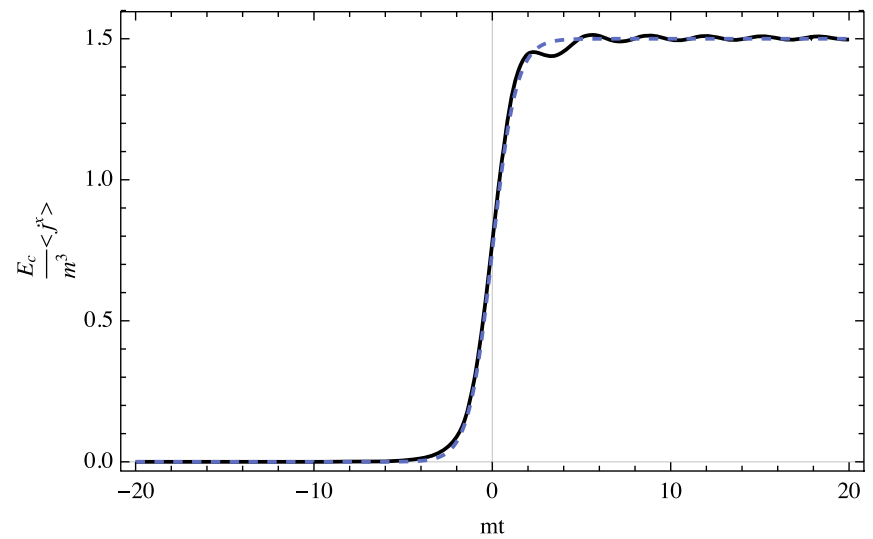

FIG. 3. $\left\langle j^{x}\right\rangle_{\text {ren }}$ for $E_{0}=4 E_{c}$ and $\omega_{0}=0.5 m$ in the case of numerical calculation (dashed line) and analytic function (black line). 


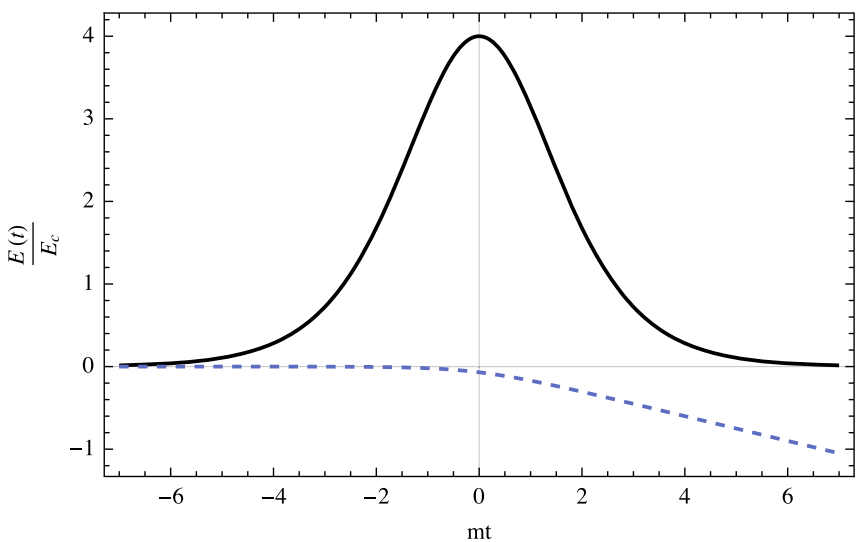

FIG. 4. Original electric field $E(t)$ (continuous line) and the electric field produced by particle creation (dashed line) in the case of $E_{0}=4 E_{c}, \omega_{0}=0.5 m$ and a coupling of $q^{2} / m^{2}=0.1$

$$
E_{b r}(t)=\frac{m^{2}}{E_{c}}[-\log \{(2 \cosh (K m t)\}-K m t]
$$

where the integration constant is fixed in order to have vanishing potential/field at $t \rightarrow-\infty$.

The electric field produced by backreaction to the particle creation becomes relevant once the pulse is almost over, when it reaches the critical electric field $E_{c}$ (see Fig. 4). It behaves now as a lineal function in time and goes in the opposite direction as the original electric field. The backreaction is significant around $m t \approx 3$.

\section{SUMMARY}

When a quantum field is coupled to a classical, nonadiabatic time-dependent background, particles are produced. As a consequence new UV divergences in local observables emerge. In cosmological scenarios, adiabatic regularization provides a very efficient method to identify and remove the unwanted divergences. In the case of an electric field background with sufficient high intensity, pairs of particles and antiparticles are produced via the Schwinger effect. Equivalent to gravitational particle production, this generates divergences in quadratic observables such as the energy momentum tensor $\left\langle T_{\mu \nu}\right\rangle$ or the electric current $\left\langle j^{\mu}\right\rangle$ and a renormalization mechanism is needed in order to predict finite quantities.

In this paper we have improved the adiabatic regularization method to include homogeneous electric fields. Our extension of the method has two folds. On the one hand, we have reexamined the method to deal with both electric and gravitational fields on an equal footing and for a quantized scalar field. In doing this we have fixed an inherent ambiguity of the method. The adiabatic order assignment of the vector potential has been traditionally assumed in the literature of zero order. Here we have argued that the correct adiabatic order assignment is one, instead of zero, at least if a gravitational field is present. This problem has been fixed by invoking the conformal anomaly. On the other hand, we have extended the adiabatic method to deal with fermions in two-dimensions. We have checked the consistency of our method by reproducing both the axial and conformal anomalies. We have also shown that only the adiabatic order assignment one for $A$ yields the right expressions for the anomalies when gravity is present. One of the main advantages of the adiabatic method in the capability to perform numerical computations. To briefly illustrate how to deal with the renormalized electric current predicted by our method we have analyzed numerically the electric current induced by a Sauter pulse.

\section{ACKNOWLEDGMENTS}

We thank F. Barbero, S. Pla, A. del Rio, F. Torrenti and E. Villaseñor for useful discussions. This work is partially funded by the Grant No. FIS2014-57387-C3-1-P, the COST action CA15117 (CANTATA), supported by COST (European Cooperation in Science and Technology), and the Severo Ochoa Program SEV-2014-0398. A. F. is supported by the Severo Ochoa Ph.D. fellowship SEV-20140398-16-1 and the European Social Fund.

\section{APPENDIX A: ADIABATIC EXPANSION WITH $A(t)$ ASSUMED OF ADIABATIC ORDER ONE}

In this appendix we provide the terms of the adiabatic expansion of the scalar field modes, up to fourth order, not included in the main text. The third order terms are

$$
\begin{aligned}
\omega^{(3)}= & -\frac{k_{x}^{3} q^{3} A^{3}}{2 a^{6} \omega^{5}}+\frac{k_{x} q^{3} A^{3}}{2 a^{4} \omega^{3}}+\frac{k_{x} q \ddot{A}}{4 a^{2} \omega^{3}}-\frac{5 k_{x} q \dot{A} \dot{\omega}}{4 a^{2} \omega^{4}}-\frac{3 k_{x} q A \ddot{\omega}}{4 a^{2} \omega^{4}}+\frac{19 k_{x} q A \dot{\omega}^{2}}{8 a^{2} \omega^{5}}+\frac{3 k_{x} \xi q A \dot{a}^{2}}{a^{4} \omega^{3}} \\
& +\frac{9 k_{x} q A \dot{a}^{2}}{8 a^{4} \omega^{3}}+\frac{3 k_{x} \xi q A \ddot{a}}{a^{3} \omega^{3}}-\frac{5 k_{x} q A \ddot{a}}{4 a^{3} \omega^{3}}-\frac{k_{x} q \dot{a} \dot{A}}{a^{3} \omega^{3}}+\frac{5 k_{x} q A \ddot{a} \dot{\omega}}{2 a^{3} \omega^{4}}, \\
H^{(3)}= & \frac{15 k_{x}^{3} q^{3} A^{3}}{16 a^{6} \omega^{13 / 2}}-\frac{5 k_{x} q^{3} A^{3}}{8 a^{4} \omega^{9 / 2}}-\frac{k_{x} q \ddot{A}}{8 a^{2} \omega^{9 / 2}}+\frac{5 k_{x} q \dot{A} \dot{\omega}}{8 a^{2} \omega^{11 / 2}}+\frac{9 k_{x} q A \ddot{\omega}}{16 a^{2} \omega^{11 / 2}}-\frac{47 k_{x} q A \dot{\omega}^{2}}{32 a^{2} \omega^{13 / 2}}-\frac{15 k_{x} \xi q A \dot{a}^{2}}{4 a^{4} \omega^{9 / 2}} \\
& -\frac{9 k_{x} q \dot{a}^{2}}{32 a^{4} \omega^{9 / 2}}-\frac{15 k_{x} \xi q A \ddot{a}}{4 a^{3} \omega^{9 / 2}}+\frac{19 k_{x} q A \ddot{a}}{16 a^{3} \omega^{9 / 2}}+\frac{k_{x} q \dot{a} \dot{A}}{2 a^{3} \omega^{9 / 2}}-\frac{5 k_{x} q A \dot{a} \dot{\omega}}{4 a^{3} \omega^{11 / 2}} .
\end{aligned}
$$


Finally, the fourth-order terms are

$$
\begin{aligned}
& \omega^{(4)}=-\frac{q^{4} A^{4}}{8 a^{4} \omega^{3}}+\frac{3 k_{x}^{2} q^{4} A^{4}}{4 a^{6} \omega^{5}}-\frac{5 k_{x}^{4} q^{4} A^{4}}{8 a^{8} \omega^{7}}-\frac{9 q^{2} \dot{a}^{2} A^{2}}{16 a^{4} \omega^{3}}-\frac{3 q^{2} \xi \dot{a}^{2} A^{2}}{2 a^{4} \omega^{3}}+\frac{103 k_{x}^{2} q^{2} \dot{a}^{2} A^{2}}{16 a^{6} \omega^{5}}+\frac{9 k_{x}^{2} q^{2} \xi \dot{a}^{2} A^{2}}{2 a^{6} \omega^{5}} \\
& -\frac{19 q^{2} \dot{\omega}^{2} A^{2}}{16 a^{2} \omega^{5}}+\frac{145 k_{x}^{2} q^{2} \dot{\omega}^{2} A^{2}}{16 a^{4} \omega^{7}}-\frac{5 q^{2} \dot{a} \dot{\omega} A^{2}}{4 a^{3} \Omega^{4}}+\frac{25 k_{x}^{2} q^{2} \dot{a} \dot{\omega} A^{2}}{2 a^{5} \omega^{6}}+\frac{5 q^{2} \ddot{a} A^{2}}{8 a^{3} \omega^{3}}-\frac{3 q^{2} \xi \ddot{a} A^{2}}{2 a^{3} \omega^{3}}-\frac{21 k_{x}^{2} q^{2} \ddot{a} A^{2}}{8 a^{5} \omega^{5}} \\
& +\frac{9 k_{x}^{2} q^{2} \xi \ddot{a} A^{2}}{2 a^{5} \omega^{5}}+\frac{3 q^{2} \ddot{\omega} A^{2}}{8 a^{2} \omega^{4}}-\frac{15 k_{x}^{2} q^{2} \ddot{\omega} A^{2}}{8 a^{4} \omega^{6}}+\frac{q^{2} \dot{a} \dot{A} A}{a^{3} \omega^{3}}-\frac{11 k_{x}^{2} q^{2} \dot{a} \dot{A} A}{2 a^{5} \omega^{5}}+\frac{5 q^{2} \dot{A} \dot{\omega} A}{4 a^{2} \omega^{4}}-\frac{25 k_{x}^{2} q^{2} \dot{A} \dot{\omega} A}{4 a^{4} \omega^{6}} \\
& -\frac{q^{2} \ddot{A} A}{4 a^{2} \omega^{3}}+\frac{3 k_{x}^{2} q^{2} \ddot{A} A}{4 a^{4} \omega^{5}}-\frac{9 \xi^{2} \dot{a}^{4}}{2 a^{4} \omega^{3}}-\frac{27 \xi \dot{a}^{4}}{8 a^{4} \omega^{3}}+\frac{63 \dot{a}^{4}}{128 a^{4} \omega^{3}}-\frac{297 \dot{\omega}^{4}}{128 \omega^{7}}-\frac{q^{2} \dot{A}^{2}}{4 a^{2} \omega^{3}}+\frac{5 k_{x}^{2} q^{2} \dot{A}^{2}}{8 a^{4} \omega^{5}}-\frac{57 \xi \dot{a}^{2} \dot{\omega}^{2}}{8 a^{2} \omega^{5}} \\
& +\frac{57 \dot{a}^{2} \dot{\omega}^{2}}{64 a^{2} \omega^{5}}-\frac{9 \xi^{2} \ddot{a}^{2}}{2 a^{2} \omega^{3}}+\frac{3 \xi \ddot{a}^{2}}{2 a^{2} \omega^{3}}-\frac{9 \ddot{a}^{2}}{32 a^{2} \omega^{3}}-\frac{13 \ddot{\omega}^{2}}{32 \omega^{5}}-\frac{15 \xi \dot{a}^{3} \dot{\omega}}{2 a^{3} \omega^{4}}+\frac{15 \dot{a}^{3} \dot{\omega}}{16 a^{3} \omega^{4}}-\frac{9 \xi^{2} \dot{a}^{2} \ddot{a}}{a^{3} \omega^{3}}+\frac{75 \xi a^{2} \ddot{a}}{8 a^{3} \omega^{3}} \\
& -\frac{27 \dot{a}^{2} \ddot{a}}{32 a^{3} \omega^{3}}-\frac{57 \xi \dot{\omega}^{2} \ddot{a}}{8 a \omega^{5}}+\frac{57 \dot{\omega}^{2} \ddot{a}}{32 a \omega^{5}}+\frac{15 \xi \dot{a} \dot{\omega} \ddot{a}}{4 a^{2} \omega^{4}}+\frac{9 \xi \dot{a}^{2} \ddot{\omega}}{4 a^{2} \omega^{4}}-\frac{9 \dot{a}^{2} \ddot{\omega}}{32 a^{2} \omega^{4}}+\frac{99 \dot{\omega}^{2} \dot{\omega}}{32 \omega^{6}}+\frac{9 \xi \ddot{a} \ddot{\omega}}{4 a \omega^{4}}-\frac{9 \ddot{a} \ddot{\omega}}{16 a \omega^{4}} \\
& -\frac{3 \dot{a} a^{(3)}}{16 a^{2} \omega^{3}}+\frac{15 \xi \dot{\omega} \dddot{a}}{4 a \omega^{4}}-\frac{15 \dot{\omega} \dddot{a}}{16 a \omega^{4}}-\frac{5 \dot{\omega} \dddot{\omega}}{8 \omega^{5}}-\frac{3 \xi \dddot{a}}{4 a \omega^{3}}+\frac{3 a^{(4)}}{16 a \omega^{3}}+\frac{\dddot{\omega}}{16 \omega^{4}} \text {, } \\
& H^{(4)}=\frac{5 q^{4} A^{4}}{32 a^{4} \omega^{9 / 2}}-\frac{45 k_{x}^{2} q^{4} A^{4}}{32 a^{6} \omega^{13 / 2}}+\frac{195 k_{x}^{4} q^{4} A^{4}}{128 a^{8} \omega^{17 / 2}}+\frac{9 q^{2} \dot{a}^{2} A^{2}}{64 a^{4} \omega^{9 / 2}}+\frac{15 q^{2} \xi \dot{a}^{2} A^{2}}{8 a^{4} \omega^{9 / 2}}-\frac{457 k_{x}^{2} q^{2} \dot{a}^{2} A^{2}}{128 a^{6} \omega^{13 / 2}}-\frac{135 k_{x}^{2} q^{2} \xi \dot{a}^{2} A^{2}}{16 a^{6} \omega^{13 / 2}} \\
& +\frac{47 q^{2} \dot{\omega}^{2} A^{2}}{64 a^{2} \omega^{13 / 2}}-\frac{871 k_{x}^{2} q^{2} \dot{\omega}^{2} A^{2}}{128 a^{4} \omega^{17 / 2}}+\frac{5 q^{2} \dot{a} \dot{\omega} A^{2}}{8 a^{3} \omega^{11 / 2}}-\frac{65 k_{x}^{2} q^{2} \dot{a} \dot{\omega} A^{2}}{8 a^{5} \omega^{15 / 2}}-\frac{19 q^{2} \ddot{a} A^{2}}{32 a^{3} \omega^{9 / 2}}+\frac{15 q^{2} \xi \ddot{a} A^{2}}{8 a^{3} \omega^{9 / 2}}+\frac{207 k_{x}^{2} q^{2} \ddot{a} A^{2}}{64 a^{5} \omega^{13 / 2}} \\
& -\frac{135 k_{x}^{2} q^{2} \xi \ddot{a} A^{2}}{16 a^{5} \omega^{13 / 2}}-\frac{9 q^{2} \ddot{\omega} A^{2}}{32 a^{2} \omega^{11 / 2}}+\frac{117 k_{x}^{2} q^{2} \ddot{\omega} A^{2}}{64 a^{4} \omega^{15 / 2}}-\frac{q^{2} \dot{a} \dot{A} A}{2 a^{3} \omega^{9 / 2}}+\frac{7 k_{x}^{2} q^{2} \dot{a} \dot{A} A}{2 a^{5} \omega^{13 / 2}}-\frac{5 q^{2} \dot{A} \dot{\omega} A}{8 a^{2} \omega^{11 / 2}}+\frac{65 k_{x}^{2} q^{2} \dot{A} \dot{\omega} A}{16 a^{4} \omega^{15 / 2}} \\
& +\frac{q^{2} \ddot{A} A}{8 a^{2} \omega^{9 / 2}}-\frac{9 k_{x}^{2} q^{2} \ddot{A} \dot{A}}{16 a^{4} \omega^{13 / 2}}+\frac{45 \xi^{2} \dot{a}^{4}}{8 a^{4} \Omega^{9 / 2}}+\frac{27 \xi \dot{a}^{4}}{32 a^{4} \omega^{9 / 2}}-\frac{99 \dot{a}^{4}}{512 a^{4} \omega^{9 / 2}}+\frac{621 \dot{\omega}^{4}}{512 \omega^{17 / 2}}+\frac{q^{2} \dot{A}^{2}}{8 a^{2} \omega^{9 / 2}}-\frac{5 k_{x}^{2} q^{2} \dot{A}^{2}}{16 a^{4} \omega^{13 / 2}} \\
& +\frac{141 \xi \dot{a}^{2} \dot{\omega}^{2}}{32 a^{2} \omega^{13 / 2}}-\frac{141 \dot{a}^{2} \dot{\omega}^{2}}{256 a^{2} \omega^{13 / 2}}+\frac{45 \xi^{2} \ddot{a}^{2}}{8 a^{2} \omega^{9 / 2}}-\frac{39 \xi \ddot{a}^{2}}{16 a^{2} \omega^{9 / 2}}+\frac{45 \ddot{a}^{2}}{128 a^{2} \omega^{9 / 2}}+\frac{29 \ddot{\omega}^{\prime 2}}{128 \omega^{13 / 2}}+\frac{15 \xi \dot{a}^{3} \dot{\omega}}{4 a^{3} \omega^{11 / 2}}-\frac{15 \dot{a}^{3} \omega}{32 a^{3} \omega^{11 / 2}} \\
& +\frac{45 \xi^{2} \dot{a}^{2} \ddot{a}}{4 a^{3} \omega^{9 / 2}}-\frac{231 \xi \dot{a}^{2} \ddot{a}}{32 a^{3} \omega^{9 / 2}}+\frac{81 \dot{a}^{2} \ddot{a}}{128 a^{3} \omega^{9 / 2}}+\frac{141 \xi \dot{\omega}^{2} \ddot{a}}{32 a \omega^{13 / 2}}-\frac{141 \dot{\omega}^{2} \ddot{a}}{128 a \omega^{13 / 2}}-\frac{15 \xi \dot{a} \dot{\omega} \ddot{a}}{8 a^{2} \omega^{11 / 2}}-\frac{27 \xi \dot{a}^{2} \ddot{\omega}}{16 a^{2} \omega^{11 / 2}}+\frac{27 \dot{a}^{2} \ddot{\omega}}{128 a^{2} \omega^{11 / 2}}-\frac{207 \dot{\omega}^{2} \ddot{\omega}}{128 \omega^{15 / 2}} \\
& -\frac{27 \xi \ddot{a} \ddot{\omega}}{16 a \omega^{11 / 2}}+\frac{27 \ddot{a} \ddot{\omega}}{64 a \omega^{11 / 2}}+\frac{3 \dot{a} \ddot{a}}{32 a^{2} \omega^{9 / 2}}-\frac{15 \xi \dot{\omega} \dddot{a}}{8 a \omega^{11 / 2}}+\frac{15 \dot{\omega} \dddot{a}}{32 a \omega^{11 / 2}}+\frac{5 \dot{\omega} \dddot{\omega}}{16 \omega^{13 / 2}}+\frac{3 \xi \dddot{a}}{8 a \omega^{9 / 2}}-\frac{3 \dddot{a}}{32 a \omega^{9 / 2}}-\frac{\dddot{\omega}}{32 \omega^{11 / 2}} .
\end{aligned}
$$

\section{APPENDIX B: ADIABATIC EXPANSION WITH $A(t)$ ASSUMED OF ADIABATIC ORDER ZERO}

The adiabatic expansion of the modes is then

$$
\begin{gathered}
\omega^{(0)}=\sqrt{\omega^{2}-\frac{2 q k_{x} A}{a^{2}}+\frac{q^{2} A^{2}}{a^{2}}} \equiv \Omega \quad H^{(0)}=\frac{1}{\omega^{(0)}} \\
\omega^{(1)}=0 \quad H^{(1)}=0 \\
\omega^{(3)}=0 \quad H^{(3)}=0 \\
H^{(2)=\frac{-6 a \Omega^{2} \ddot{a}-3 \Omega^{2} \dot{a}^{2}-2 a^{2} \Omega \ddot{\Omega}+3 a^{2} \Omega^{2}}{16 a^{2} \Omega^{9 / 2}}} \quad \omega^{(2)}=-\frac{2 a \Omega^{2} \ddot{a}-\Omega^{2} \dot{a}^{2}+2 a^{2} \Omega \ddot{\Omega}-3 a^{2} \dot{\Omega}^{2}}{8 a^{2} \Omega^{3}}
\end{gathered}
$$




$$
\begin{aligned}
H^{(4)}= & \frac{3 \xi \dddot{a}}{8 a \Omega^{9 / 2}}-\frac{3 \dddot{a}}{32 a \Omega^{9 / 2}}-\frac{15 \xi \dddot{a} \dot{\Omega}}{8 a \Omega^{11 / 2}}+\frac{15 \dddot{a} \dot{\Omega}}{32 a \Omega^{11 / 2}}+\frac{45 \xi^{2} \ddot{a}^{2}}{8 a^{2} \Omega^{9 / 2}}-\frac{27 \xi \ddot{a} \ddot{\Omega}}{16 a \Omega^{11 / 2}}+\frac{141 \xi \ddot{a} \dot{\Omega}^{2}}{32 a \Omega^{13 / 2}}-\frac{39 \xi \ddot{a}^{2}}{16 a^{2} \Omega^{9 / 2}}+\frac{27 \ddot{a} \ddot{\Omega}}{64 a \Omega^{11 / 2}} \\
& -\frac{141 \ddot{a} \ddot{\Omega}^{2}}{128 a \Omega^{13 / 2}}+\frac{45 \ddot{a}^{2}}{128 a^{2} \Omega^{9 / 2}}+\frac{45 \xi^{2} \dot{a}^{4}}{8 a^{4} \Omega^{9 / 2}}-\frac{27 \xi \dot{a}^{2} \ddot{\Omega}}{16 a^{2} \Omega^{11 / 2}}+\frac{15 \xi \dot{a}^{3} \dot{\Omega}}{4 a^{3} \Omega^{11 / 2}}+\frac{141 \xi \dot{a}^{2} \dot{\Omega}^{2}}{32 a^{2} \Omega^{13 / 2}}+\frac{27 \xi \dot{a}^{4}}{32 a^{4} \Omega^{9 / 2}}+\frac{27 \dot{a}^{2} \ddot{\Omega}}{128 a^{2} \Omega^{11 / 2}} \\
& -\frac{15 \dot{a}^{3} \dot{\Omega}}{32 a^{3} \Omega^{11 / 2}}-\frac{141 \dot{a}^{2} \dot{\Omega}^{2}}{256 a^{2} \Omega^{13 / 2}}-\frac{99 \dot{a}^{4}}{512 a^{4} \Omega^{9 / 2}}+\frac{3 a^{(3)} \dot{a}}{32 a^{2} \Omega^{9 / 2}}+\frac{45 \xi^{2} \dot{a}^{2} \ddot{a}}{4 a^{3} \Omega^{9 / 2}}-\frac{15 \xi \dot{a} \ddot{a} \dot{\Omega}}{8 a^{2} \Omega^{11 / 2}}-\frac{231 \xi \dot{a}^{2} \ddot{a}}{32 a^{3} \Omega^{9 / 2}}+\frac{81 \dot{a}^{2} \ddot{a}}{128 a^{3} \Omega^{9 / 2}}-\frac{\dddot{\Omega}}{32 \Omega^{11 / 2}} \\
& +\frac{29 \ddot{\Omega}^{2}}{128 \Omega^{13 / 2}}+\frac{621 \dot{\Omega}^{4}}{512 \Omega^{17 / 2}}+\frac{5 \dddot{\Omega} \dot{\Omega}}{16 \Omega^{13 / 2}}-\frac{207 \dot{\Omega}^{2} \ddot{\Omega}}{128 \Omega^{15 / 2}} \\
\omega^{(4)}= & -\frac{3 \xi \dddot{a}}{4 a \Omega^{3}}+\frac{3 \dddot{a}}{16 a \Omega^{3}}+\frac{15 \xi \ddot{a} \dot{\Omega}}{4 a \Omega^{4}}-\frac{15 \dddot{a} \dot{\Omega}}{16 a \Omega^{4}}-\frac{9 \xi^{2} \ddot{a}^{2}}{2 a^{2} \Omega^{3}}+\frac{3 \xi \ddot{a}^{2}}{2 a^{2} \Omega^{3}}+\frac{9 \xi \ddot{a} \Omega}{4 a \Omega^{4}}-\frac{57 \xi \ddot{a} \dot{\Omega}^{2}}{8 a \Omega^{5}}-\frac{9 \ddot{a}^{2}}{32 a^{2} \Omega^{3}}-\frac{9 \ddot{a} \ddot{\Omega}}{16 a \Omega^{4}}+\frac{57 \ddot{a} \dot{\Omega}^{2}}{32 a \Omega^{5}} \\
& -\frac{27 \xi \dot{a}^{4}}{8 a^{4} \Omega^{3}}-\frac{9 \xi^{2} \dot{a}^{4}}{2 a^{4} \Omega^{3}}+\frac{63 \dot{a}^{4}}{128 a^{4} \Omega^{3}}-\frac{15 \xi \dot{a}^{3} \dot{\Omega}}{2 a^{3} \Omega^{4}}+\frac{15 \dot{a}^{3} \dot{\Omega}}{16 a^{3} \Omega^{7} 4}+\frac{9 \xi \dot{a}^{2} \ddot{\Omega}}{4 a^{2} \Omega^{4}}+\frac{15 \xi \dot{a} \ddot{a} \dot{\Omega}^{2}}{4 a^{2} \Omega^{4}}-\frac{57 \xi \dot{a}^{2} \dot{\Omega}^{2}}{8 a^{2} \Omega^{5}}-\frac{9 \dot{a}^{2} \ddot{\Omega}}{32 a^{2} \Omega^{4}}+\frac{57 \dot{a}^{2} \dot{\Omega}^{2}}{64 a^{2} \Omega^{5}} \\
& -\frac{9 \xi^{2} \dot{a}^{2} \ddot{a}}{a^{3} \Omega^{3}}+\frac{75 \xi \dot{a}^{2} \ddot{a}}{8 a^{3} \Omega^{3}}-\frac{27 \dot{a}^{2} \ddot{a}}{32 a^{3} \Omega^{3}}-\frac{3 \dddot{a} \dot{a}}{16 a^{2} \Omega^{3}}+\frac{\dddot{\Omega}}{16 \Omega^{4}}-\frac{13 \ddot{\Omega}^{2}}{32 \Omega^{5}}-\frac{297 \dot{\Omega}^{4}}{128 \Omega^{7}}-\frac{5 \dddot{\Omega} \dot{\Omega}}{8 \Omega^{5}}+\frac{99 \dot{\Omega}^{2} \ddot{\Omega}}{32 \Omega^{6}} .
\end{aligned}
$$

[1] L. Parker and D. J. Toms, Quantum Field Theory in Curved Spacetime: Quantized Fields and Gravity (Cambridge University Press, Cambridge, England, 2009).

[2] R. M. Wald, Quantum Field Theory in Curved Space-time and Black Hole Thermodynamics (University of Chicago Press, Chicago, 1994).

[3] S. Fulling, Aspects of Quantum Field Theory in Curved Space-Time (Cambridge University Press, Cambridge, England, 1989).

[4] N. D. Birrell and P. C.W. Davies, Quantum Fields in Curved Space (Cambridge University Press, Cambridge, England, 1982).

[5] L. Parker, The creation of particles in an expanding universe, Ph. D. thesis, Harvard University, 1966. Dissexpress.umi.com, Publication Number 7331244.

[6] L. Parker, Phys. Rev. Lett. 21, 562 (1968); Phys. Rev. D 183, 1057 (1969); 3, 346 (1971).

[7] S. A. Fulling, Phys. Rev. D 7, 2850 (1973).

[8] S. W. Hawking, Nature (London) 248, 30 (1974); Commun. Math. Phys. 43, 199 (1975).

[9] L. H. Ford and L. Parker, Phys. Rev. D 16, 1601 (1977).

[10] L. P. Grishchuk, Zh. Eksp. Teor. Fiz 67, 825 (1974) [Sov. Phys. JETP 40, 409 (1975)]; Ann. NY Acad. Sci. 302, 439 (1977).

[11] A. H. Guth, Phys. Rev. D 23, 347 (1981).

[12] V. F. Mukhanov and G. V. Chibisov, JETP Lett. 33, 532 (1981); Pis'ma Zh. Eksp. Teor. Fiz. 33, 549 (1981); S. W. Hawking, Phys. Lett. 115B, 295 (1982); A. Guth and S.-Y. Pi, Phys. Rev. Lett. 49, 1110 (1982); A. A. Starobinsky, Phys. Lett. 117B, 175 (1982); J. M. Bardeen, P. J. Steinhardt, and M.S. Turner, Phys. Rev. D 28, 679 (1983).
[13] L. Kofman, A. Linde, and A. Starobinsky, Phys. Rev. Lett. 73, 3195 (1994); Phys. Rev. D 56, 3258 (1997).

[14] The Extreme Light Infrastructure (ELI) project: www .extreme-light-infrastructure.eu/.

[15] G. V. Dunne and G. V. Dunne, Int. J. Mod. Phys. A27, 1260004 (2012); Eur. Phys. J. D 55, 327 (2009); B. S. Xie, Z. L. Lie, and S. Tang, Matter and Radiation at Extremes, 2, 225 (2017).

[16] W. Pittrich and H. Gies, Probing the Quantum Vacuum (Springer, Heidelberg, 2000).

[17] J. Schwinger, Phys. Rev. 82, 664 (1951).

[18] F. Sauter, Z. Phys. 69, 742 (1931).

[19] W. Heisenberg and H. Euler, Z. Phys. 98, 714 (1936).

[20] E. Brezin and C. Itzykson, Phys. Rev. D 2, 1191 (1970).

[21] V. S. Popov, Zh. Eksp. Teor. Fiz. 62, 1248 (1972) [Sov. Phys. JETP 34, 709 (1972)].

[22] G. V. Dunne, Heisenberg-Euler effective Lagrangians: Basics and extensions, in I. Kogan Memorial Volume, From Fields to Strings: Circumnavigating Theoretical Physics, edited by M. Shifman, A. Vainshtein, and J. Wheater (World Scientific, Singapore, 2005).

[23] G. Dunne and T. Hall, Phys. Rev. D 58, 105022 (1998).

[24] R. Schutzhold, H. Gies, and G. Dunne, Phys. Rev. Lett. 101, 130404 (2008).

[25] A. Di Piazza, E. Lotstedt, A. I. Milstein, and C. H. Keitel, Phys. Rev. Lett. 103, 170403 (2009).

[26] F. Hebenstreit, R. Alkofer, G. V. Dunne, and H. Gies, Phys. Rev. Lett. 102, 150404 (2009); C. K. Dumlu and G. V. Dunne, Phys. Rev. Lett. 104, 250402 (2010); E. Akkermans and G. V. Dunne, Phys. Rev. Lett. 108, 030401 (2012).

[27] T. C. Adorno, S. P. Gavrilov, and D. M. Gitman, Phys. Scripta 90, 074005 (2015). 
[28] T. C. Adorno, S. P. Gavrilov, and D. M. Gitman, Eur. Phys. J. C 76, 447 (2016).

[29] T. C. Adorno, S. P. Gavrilov, and D. M. Gitman, Int. J. Mod. Phys. A 32, 1750105 (2017).

[30] L. Parker and S. A. Fulling, Phys. Rev. D 9, 341 (1974); S. A. Fulling and L. Parker, Ann. Phys. (N.Y.) 87, 176 (1974); S. A. Fulling, L. Parker, and B. L. Hu, Phys. Rev. D 10, 3905 (1974).

[31] T. S. Bunch, J. Phys. A 13, 1297 (1980).

[32] P. R. Anderson and L. Parker, Phys. Rev. D 36, 2963 (1987); P. R. Anderson and W. Eaker, Phys. Rev. D 61, 024003 (1999); S. Habib, C. Molina-Paris, and E. Mottola, Phys. Rev. D 61, 024010 (1999).

[33] A. Landete, J. Navarro-Salas, and F. Torrenti, Phys. Rev. D 88, 061501(R) (2013); 89, 044030 (2014).

[34] A. del Rio, J. Navarro-Salas, and F. Torrenti, Phys. Rev. D 90, 084017 (2014).

[35] S. Ghosh, Phys. Rev. D 91, 124075 (2015); 93, 044032 (2016).

[36] A. del Rio, A. Ferreiro, J. Navarro-Salas, and F. Torrenti, Phys. Rev. D 95, 105003 (2017).

[37] T. Kobayashi and N. Afshordi, J. High Energy Phys. 10 (2014) 166.

[38] C. Stahl, E. Strobel, and S.-S. Xue, Phys. Rev. D 93, 025004 (2016).

[39] I. Agullo, A. Landete, and J. Navarro-Salas, Phys. Rev. D 90, 124067 (2014); A. del Rio and J. Navarro-Salas, Phys. Rev. D 91, 064031 (2015); I. Agullo, J. Navarro-Salas, G. J. Olmo, and L. Parker, Phys. Rev. Lett. 103, 061301 (2009); Phys. Rev. D 81, 043514 (2010).
[40] F. Cooper and E. Mottola, Phys. Rev. D 40, 456 (1989).

[41] Y. Kluger, J. M. Eisenberg, B. Svetitsky, F. Cooper, and E. Mottola, Phys. Rev. Lett. 67, 2427 (1991).

[42] Y. Kluger, J. M. Eisenberg, B. Svetitsky, F. Cooper, and E. Mottola, Phys. Rev. D 45, 4659 (1992).

[43] Y. Kluger, J. M. Eisenberg, and B. Svetitsky, Int. J. Mod. Phys. E 02, 333 (1993).

[44] P. Anderson and E. Mottola, Phys. Rev. D 89, 104039 (2014).

[45] J. F. Donoghue and B. K. El-Menoufi, J. High Energy Phys. 05 (2015) 118.

[46] R. A. Bertlmann, Anomalies in Quantum Field Theory (Oxford University Press, New York, 2000).

[47] A. S. Blaer, N. H. Christ, and J.-F. Tang, Phys. Rev. Lett. 47, 1364 (1981).

[48] I. Agullo, A. del Rio, and J. Navarro-Salas, Int. J. Mod. Phys. D 26, 1742001 (2017); Phys. Rev. Lett. 118, 111301 (2017).

[49] M. J. Duff, Nucl. Phys. B125, 334 (1977).

[50] P. C. W. Davies and W. G. Unruh, Proc. R. Soc. A 356, 259 (1977).

[51] M. J. Duff, Classical Quantum Gravity 11, 1387 (1994).

[52] A. Erdelyi, W. Magnus, F. Oberhettinger, and F. Tricomi, Higher transcendental functions (McGraw-Hill Book Company, New York, 1953), Vol. I.

[53] Higher Transcendental Functions (Bateman Manuscript Project), edited by A. Erdelyi (McGraw-Hill, New York, 1953; Nauka, Moscow, 1980; Pergamon, Oxford, 1982), Vol 2.

[54] A. I. Nikishov, Theor. Math. Phys. 136, 958 (2003). 